Alexander Oeser*, Jan Gaebel and Thomas Kuhnt

\title{
Development of an Assistance System for the Intuitive Assessment of Laboratory Findings in Oncology
}

\begin{abstract}
Due to the broad spectrum of individual functionalities, current hospital information systems in most cases do not offer quick information access or proper assistance in information assessment. This results in extensive use of raw value representations and the need to process and evaluate those cognitively through the physician. This can result in an error-prone and ineffective process, especially in complex and chronic treatment scenarios. With an example scenario of laboratory value assessment in head and neck oncology, we have evaluated the requirements for extensive support of case interpretation through the conduction of a Delphi study including five medical experts with different levels of expertise. Based on the results, we propose a solution with integrated HL7 FHIR compliant clinical decision-support functionality through a set of integrated rules from relevant clinical practice guidelines. Furthermore, we introduce the implementation of a dedicated application that allows for the definition of custom laboratory value evaluation rules to enable automated and patientindividual value interpretation.
\end{abstract}

Keywords: Clinical Decision Support, Laboratory Findings, Clinical Knowledge Management, HL7 FHIR, Clinical Assistance, Head and Neck Oncology

https://doi.org/10.1515/cdbme-2019-0016

\section{Background and Significance}

The landscape of clinical IT systems is characterized by a diverse set of specialized applications. Apart from embedded hardware- or domain-specific solutions, that are tailored for one specific use-case, hospital information systems (HIS) that manage a patient's Electronic Medical Record (EMR) comprise a much broader feature-set and aim to support various aspects of the clinical routine. While common HIS offer integrated viewers for laboratory findings, they often only present raw numeric values with limited assistance features (e.g. basic color-coding or simple deviation indicators from the reference ranges). However, those features are tied to individual measures and do not take into account overarching patient characteristics like disease types or medication that might naturally impact the extent of a certain value. Thus, each report needs to be processed and evaluated cognitively by the respective user, which can be error-prone and ineffective [1].

\subsection{Clinical use-case}

In the case of long-term treatment strategies, e.g. radiochemotherapies in oncology, the allocation of scheduled doses needs to be assessed before every treatment session. During this procedure, the patient's health status and therapytolerance are evaluated through a set of laboratory measures. Due to extensive collection of data in chronic disease management, relevant information from the patient's EMR can easily become hard to assess for the physician, which introduces problems related to patient safety (e.g. oversights) [2].

\subsection{Utilization of health information technologies}

Brailer et al. have defined health information technologies (HIT) as "the application of information processing involving both computer hardware and software that deals with the

\footnotetext{
*Corresponding author: Alexander Oeser: Innovation Center Computer Assisted Surgery (ICCAS), Semmelweisstrasse 14, Leipzig, Germany, e-mail: alexander.oeser@medizin.unileipzig.de Jan Gaebel: Innovation Center Computer Assisted Surgery (ICCAS), Leipzig, Germany

Thomas Kuhnt: University of Leipzig - Medical Center Department of Radiooncology, Leipzig, Germany
} 


\section{DE GRUYTER}

Alexander Oeser et al., Development of an Assistance System for the Intuitive Assessment of Laboratory Findings in - 62

storage, retrieval, sharing, and use of health care information, data, and knowledge for communication and decision making" [6]. Thus, systems utilizing HIT aim to provide assistance through the processing of available data to enable automated assessment based on formalized medical knowledge. For the presented use-case, such support might be provided by visualization. The display of laboratory findings through different types of graphical representations has been the subject of several research studies. However, their assessment by the user remains a subjective task that heavily relies upon individual taste [3] and the extent of the presented case [4]. Thus, no final statement about an optimal representation format can be made.

Another way to provide assistance in laboratory value assessment is clinical decision-support (CDS), which utilizes formalized clinical knowledge to automatically evaluate the incoming data [7]. This approach has already shown significant improvements in a variety of clinical applications $[8,9]$ as well as in laboratory medicine [10]. The fact, that CDS requires the presence of prior knowledge about the respective use-case to make automatic assessments emphasizes the process of knowledge integration, since the significance of the CDS functionality is correlated to the quality of the present knowledge-base [11].

\subsection{Objective}

To enable efficient support in the assessment of laboratory findings, we propose a novel solution that supports automatic value interpretation based on clinical practice guidelines (CPG) combined with the integration of individual evaluation rules through a dedicated editor. In this way, we aim to provide extensive support for monitoring and risk estimation during long-term treatment procedures while preventing confounding factors such as over-alerting. Although our method is not limited to a certain medical domain and can be adapted to a series of use-cases, we will demonstrate our approach in the context of head and neck oncology.

\section{Material and Methods}

To ensure that our objective aligns with the needs of physicians in the relevant departments, we have conducted a Delphi study to determine the requirements for a novel take on laboratory order assessment and to reveal the flaws of current systems. The mixed group of participants consisted of experts from the fields of medical-, radiological- and head and neck oncology at the University Hospital Leipzig, each with different levels of professional expertise (see Table 1).

Table 1: Characteristics of the participants in the Delphi study

\begin{tabular}{llr}
\hline Participant & Department & Years of Expertise \\
\hline 1 & Medical Oncology & 7 \\
2 & Radiation Oncology & 24 \\
3 & Head \& Neck Oncology & 6 \\
4 & Head \& Neck Oncology & 7 \\
5 & Head \& Neck Oncology & 13 \\
\hline
\end{tabular}

During each interview, we provided a short presentation of our objective to determine its significance for the clinical routine. The presentation was then followed by an extensive feedback cycle that invited the participants to provide a review of the proposal as well as the intended functionality. Beginning with the second participant, the received feedback from the preceding interviews was also presented to reveal the current state including all complaints and useful extensions. After the five interviews had been conducted, we provided the gathered feedback to each participant to ensure an overall consensus among all contributors.

To satisfy the requirements of our use-case, we consulted the Department of Radiooncology at the University of Leipzig Medical Center to obtain the current status-quo of data retrieval for patients undergoing cancer treatment in head and neck oncology. This resulted in a series of relevant information entities which served as the baseline of our system design (see Table 2).

Table 2: List of the relevant information entities for radio-chemotherapy monitoring in head and neck oncology

\begin{tabular}{|c|c|}
\hline Classification & Information Entities \\
\hline Hemogram & $\begin{array}{l}\text { Erythrocytes, Hemoglobin, Hematocrite, } \\
\mathrm{MCH}, \mathrm{MCHC}, \mathrm{MCV} \text {, Leucocytes, } \\
\text { Thrombocytes }\end{array}$ \\
\hline $\begin{array}{l}\text { Differential Blood } \\
\text { Count }\end{array}$ & $\begin{array}{l}\text { Leucocytes, Lymphocytes, Neutrophil } \\
\text { Granulocyte, Eosinophile Granulocytes, } \\
\text { Basophile Granulocytes }\end{array}$ \\
\hline $\begin{array}{l}\text { Other Laboratory } \\
\text { Diagnostics }\end{array}$ & $\begin{array}{l}\text { Sodium, Potassium, Chloride, } \\
\text { Magnesium, Creatinine, Urea, Uric Acid, } \\
\text { Cystatine C, CRP, ALAT, ASAT, AP, } \\
\text { Gamma-GT, Bilirubin, Cholinesterase, } \\
\text { Albumin, Total Protein, Quick, PPT, } \\
\text { Fibrinogen, TZ, TSH (basal), fT3, fT4 }\end{array}$ \\
\hline Other Diagnostics & ECG, Transesophageal Echo, Audiogram, \\
\hline
\end{tabular}




\begin{tabular}{ll}
\hline Classification & Information Entities \\
\hline Other Conditions & $\begin{array}{l}\text { Renal Scintigraphy } \\
\text { (dental) }\end{array}$ \\
\hline
\end{tabular}

While some entities require the assessment of external departments (e.g. ECG, Audiogram) and thus need to be considered as categorial values (e.g. good or bad, yes or no), the laboratory values are represented as plain numbers and are raised periodically throughout the treatment process.

To integrate an initial point for the automatic value evaluation, we've defined a set of classification rules for the list of values based on relevant CPGs. In our case, we've considered the American Board of Internal Medicine (ABIM) reference ranges on laboratory results as well as the Common Terminology Criteria for Adverse Events (CTCAE) in version 5.0 for risk classification.

\section{Results}

While the overall concept and functionality of the system was considered as suitable for the assessment of laboratory findings, the Delphi study revealed substantial aspects that derived from use-case specific requirements. Firstly, the system had to feature a visual prioritization of pathologic values to enhance focus and increase efficiency in reading the laboratory reports. Those visual accentuations should also include warnings to directly capture the user's attention. Secondly, deviations from the reference ranges of the laboratory values should feature another layer of visual distinction that is based on their significance for the case. Thirdly, the progression of a value over time should be emphasized, e.g. to reveal treatment responses.

While the integration of visual distinctions and the focus on value progression are rather simple technical problems that can be addressed through design-related considerations (e.g. color-coding and chart visualizations), the provision of case-specific value assessments and individual warnings require more sophisticated CDS mechanisms combined with formal logical expressions. Since the correct interpretation of certain values in oncologic treatment is a multi-dimensional problem, it requires the consideration of various patient characteristics simultaneously. While some automatic detections can be derived from the integration of CPGs, those rather universal and value-specific considerations facilitate the risk of over-alerting or missing out on critical conditions based on multi-value assessments.

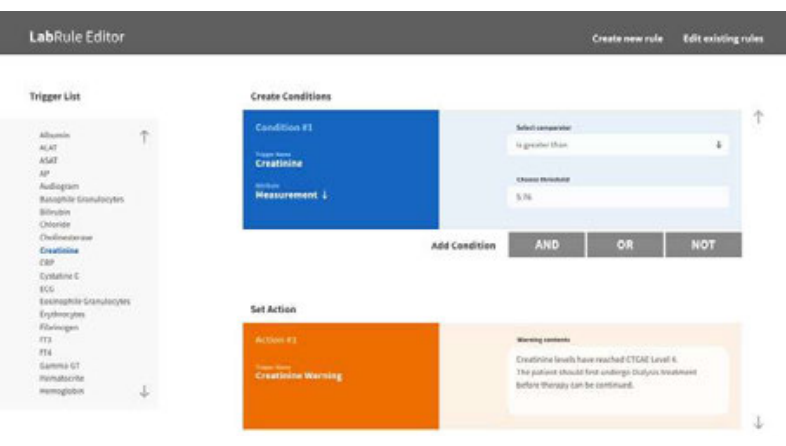

'Figure 1: Screenshot of the custom CDS rule editor

Since the possible value combinations needed for proper therapy risk estimation are extensive, we have developed a custom application that enables the intuitive construction of custom CDS rules for warnings beyond the initially integrated CPGs. This allows for an adaptation of automatic value assessment for the respective case based on individual policies within the clinical department.

The application features two dedicated components and is built on top of the HL7 FHIR specification for CDS services. This allows for the implementation of custom value evaluation rules integrating a FHIR server for patient data retrieval and CDS response handling [12]. First off, the editor component enables the physician to construct conditional blocks that can be combined through logical operators to form evaluation rules based on the available patient data (Figure 1). Those rules are then stored within a dedicated json file.

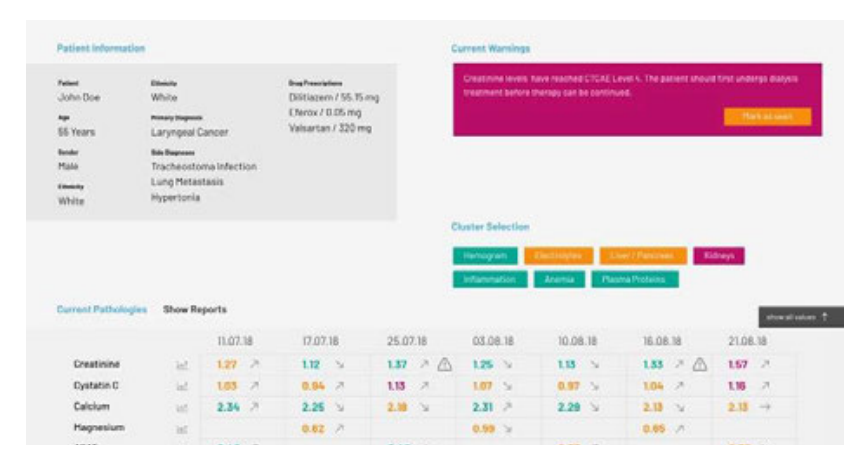

Figure 2: Screenshot of the dashboard component for intuitive value assessment

A dashboard component handles the visualization of the laboratory findings in the form of an extended digital report (Figure 2). The component comprises three distinct sections: a patient inspector featuring relevant information entities from a patient's EMR, an alert window for the chronological display of patient-specific warnings, and a dedicated container for overall value representation, either based on distinct values or value progression through an optional linechart visualization. When a patient case is invoked through 


\section{DE GRUYTER}

Alexander Oeser et al., Development of an Assistance System for the Intuitive Assessment of Laboratory Findings in - 64

the dashboard, the FHIR "patient-view" hook triggers our CDS service and is utilizing the previously stored custom rule definitions as well as the previously integrated CPGs. FHIR resources for the patient-specific conditions and laboratory measures are inserted through the "pre-fetchtemplate" parameter. After matching each value with the present evaluation rules, the responses are then returned as FHIR "cards" which contain the results of the interpretation for frontend display, e.g. the evaluation of value significance or the respective CTCAE risk classification. The dashboard integrates the Vue.js JavaScript framework to handle dynamic rendering of the user interface (UI). This allows for an integration of rendering conditions for every UI entity, which enables us to provide value-specific color-coding and alert handling during runtime. Furthermore, the UI automatically appends a dedicated icon to each value that has been evaluated by the CDS service. This allows the physician to easily distinguish if an assessment is based on default reference ranges or custom rules.

\section{Outlook and Discussion}

While the conducted Delphi study ensured a consensus-based structural and functional baseline for the project, the limited number of participants might not represent a holistic view on all possible use-cases and scenarios. Thus, the benefits of the system need to be evaluated in a prospective study related to the usability or efficiency of the actual application. Assistance systems or clinical decision support systems (CDSS) in general are an important addition to safe decisionmaking and personalized medicine. However, their calculations and presentations must be based on EMRs. Using these systems must be associated with little to no additional efforts for the physicians. To be fully integrated into the physicians' workflow, those systems must also connect well with hospital information systems. Relying on web services allows for easy integration. A system approach as proposed by Gaebel et al. might enable standardized access to clinical databases [13]. The system architecture connects different modules with the underlying information system through web services. This modular infrastructure allows for an exchange of individual modules as well as an addition of new application systems for specialized assistance, as proposed in this paper.

\section{Author Statement}

This work was funded by the German Federal Ministry of Education and Research (BMBF). The statements made herein are solely the responsibility of the authors. The authors declare that they have no conflict of interest. No formal consent is required.

\section{References}

[1] Obenaus M, Burgsteiner H. Visualization of CDA laboratory reports and long term trends as a possible EHR application for patients and physicians. Stud Health Technol Inform. 2014;198:93-100.

[2] Beasley JW, Wetterneck TB, Temte J, Lapin JA, Smith P, Rivera-Rodriguez AJ, et al. Information chaos in primary care: implications for physician performance and patient safety. J Am Board Fam Med. 2011 Dec;24(6):745-51.

[3] Bauer DT, Guerlain S, Brown PJ. The design and evaluation of a graphical display for laboratory data. J Am Med Inform Assoc. 2010 Aug;17(4):416-24.

[4] Torsvik T, Lillebo B, Mikkelsen G. Presentation of clinical laboratory results: an experimental comparison of four visualization techniques. J Am Med Inform Assoc. 2013 Apr;20(2):325-31.

[5] Torsvik T, Lillebo B, Hertzum M. How Do Experienced Physicians Access and Evaluate Laboratory Test Results for the Chronic Patient? A Qualitative Analysis. Appl Clin Inform. 2018 Apr;9(2):403-10.

[6] Thompson TG, Brailer DJ. The decade of health information technology: delivering consumer-centric and information-rich health care: framework for strategic action [Internet]. Washington, D.C: U.S. Dept. of Health and Human Services, Office for the National Coordinator for Health Information Technology; 2004 [cited 2019 May 28]. 1 p. Available from: http://purl.fdlp.gov/GPO/gpo800

[7] Osheroff JA MD, Teich JM MD, Ph D, Donald MDL, Luis MDS, Velasco FT MD. Improving Outcomes With Clinical Decision Support: An Implementer's Guide. 2nd ed. Chicago, IL: Himss Publishing; 2012. 323 p.

[8] Bates DW, Teich JM, Lee J, Seger D, Kuperman GJ, Ma'Luf $\mathrm{N}$, et al. The impact of computerized physician order entry on medication error prevention. J Am Med Inform Assoc 1999; 6:313-21.

[9] Ricciardi TN, Masarie FE, Middleton B. Clinical benchmarking enabled by the digital health record. Stud Health Technol Inform2001; 84:675-9.

[10] Carmona-Cejudo JM, Hortas ML, Baena-García M, LanaLinati J, González C, Redondo M, et al. DB4US: A Decision Support System for Laboratory Information Management. Interact J Med Res [Internet]. 2012 Nov 14 [cited 2019 May 27];1(2). Available from: https://www.ncbi.nlm.nih.gov/pmc/articles/PMC3626127/

[11] Musen MA, van der Lei J. Knowledge engineering for clinical consultation programs: modeling the application area. Methods Inf Med. 1989 Jan;28(1):28-35.

[12] FHIR.org. Clinical Decision Support - FHIR v4.0.0 [Internet]. [cited 2019 May 31]. Available from: https://www.hl7.org/fhir/clinicalreasoning-cds-on-fhir.html

[13] Gaebel J, Schreiber E, Oeser A, Oeltze-Jafra S. Modular Architecture for Integrated Model-Based Decision Support. Stud Health Technol Inform 2018; 248:108-115 